\title{
Extracting Features from Time-Dependent Vector Fields Using Internal Reference Frames
}

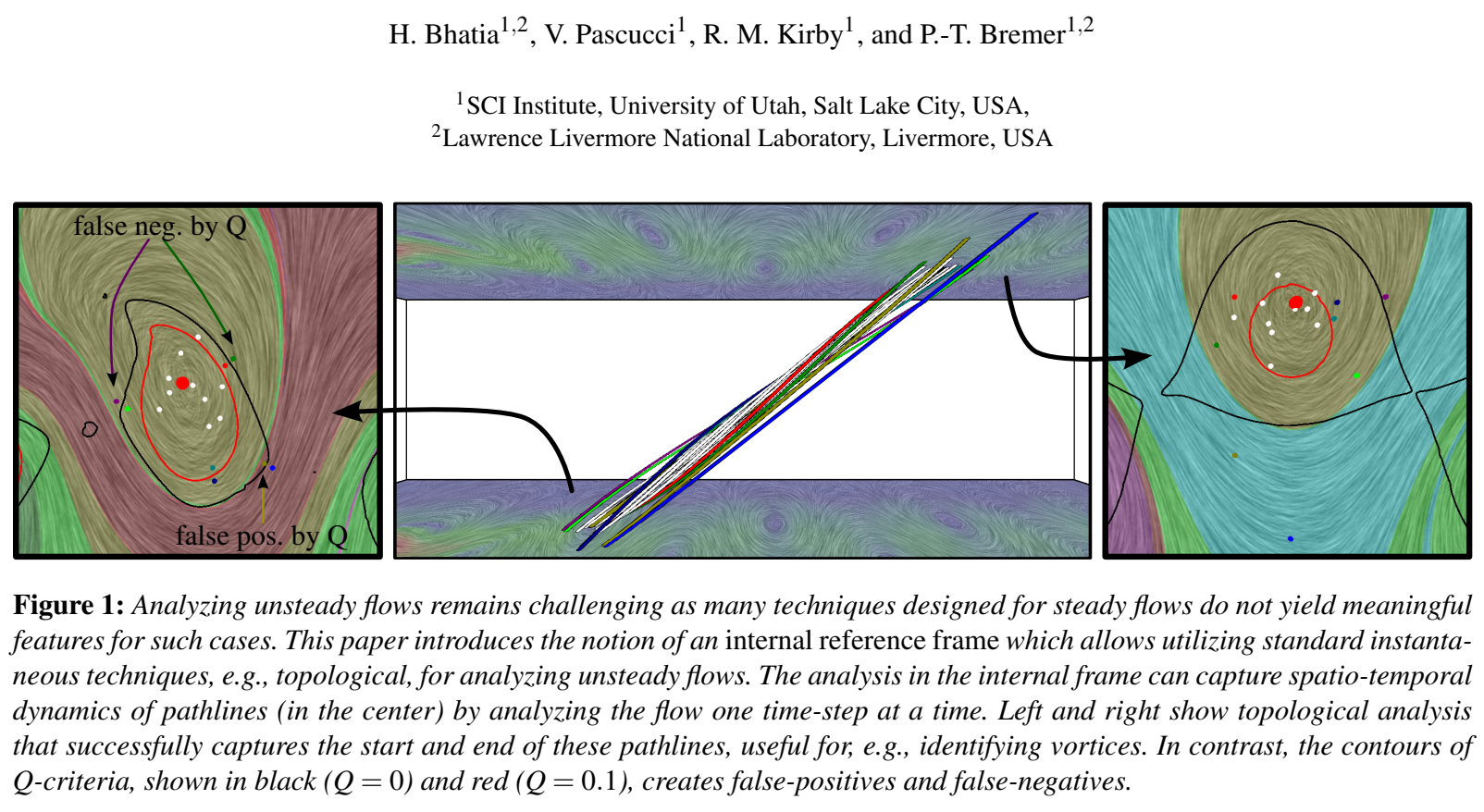

\begin{abstract}
Extracting features from complex, time-dependent flow fields remains a significant challenge despite substantial research efforts, especially because most flow features of interest are defined with respect to a given reference frame. Pathline-based techniques, such as the FTLE field, are complex to implement and resource intensive, whereas scalar transforms, such as $\lambda_{2}$, often produce artifacts and require somewhat arbitrary thresholds. Both approaches aim to analyze the flow in a more suitable frame, yet neither technique explicitly constructs one.

This paper introduces a new data-driven technique to compute internal reference frames for large-scale complex flows. More general than uniformly moving frames, these frames can transform unsteady fields, which otherwise require substantial processing of resources, into a sequence of individual snapshots that can be analyzed using the large body of steady-flow analysis techniques. Our approach is simple, theoretically well-founded, and uses an embarrassingly parallel algorithm for structured as well as unstructured data. Using several case studies from fluid flow and turbulent combustion, we demonstrate that internal frames are distinguished, result in temporally coherent structures, and can extract well-known as well as notoriously elusive features one snapshot at a time.

Categories and Subject Descriptors (according to ACM CCS): I.6.6 [Computing Methodologies]: Simulation And Modeling-Simulation Output Analysis;
\end{abstract}

\section{Introduction}

Time-dependent or unsteady flows are one of the most common forms of data in scientific and engineering simulations describing phenomena such as ocean currents [MBP10], wind patterns [BMP06], and turbulent com- bustion [GGK*12, YRSC11]. However, despite substantial research efforts in physics and visualization, analyzing such flows remains challenging. One of the fundamental problems is that unlike unsteady scalar fields, unsteady flows cannot be easily analyzed one time-step at a time. It is well 
known that such instantaneous analysis, e.g., using vector field topology [HH89], does not lead to meaningful features [Lug79, PT84, PC94]. Instead, it has been pointed out that unsteady flows must be analyzed in a reference frame, where they are steady or near-steady in order for the corresponding features to be temporally coherent and practically meaningful. Note that, the exact definition of the term reference frames in this context is subtle and often differs between communities (see Section 4.1).

The most common approach to address this problem is to study flows indirectly by computing derived Galileaninvariant scalar indicators, which are invariant to uniformly moving frames. However, these techniques, by necessity, lose a significant amount of information. In particular, intuitive features of flow are replaced by indicators, many of which have significant flaws and are known to produce artifacts. For example, common indicators to identify vortices [Hun87, JH95] are not necessarily ideal, as they can also indicate shear, are influenced by noise, and typically require a somewhat arbitrary threshold to define a vortex.

More recently, a second class of techniques has been developed, which uses the particles' frame of reference, by analyzing their pathlines, i.e., the paths along which these massless particles are advected by the flow. Computing the differences between a dense set of pathlines for a finite time interval allows one to extract the finite-time Lyapunov exponent (FTLE) [Hal00], which is then used to identify flow barriers [Hal01]. However, these pathlines are typically approximated through numerical integration, and are computationally expensive [GGTH07]. Furthermore, the choice of the correct time-scale for pathlines is non-trivial, but has a large impact on the results. Finally, by definition, such techniques require simultaneous access to a large number of time-steps at a high temporal resolution for the corresponding pathlines to accurately represent the underlying flow. Unfortunately, due to I/O bandwidth bottlenecks, it is infeasible to store the required number of time-steps for the largest and the most interesting simulations carried out on modern highperformance computers. This limits the practical usability of the pathline-based approaches. With the anticipated developments in future hardware, this problem is only expected to worsen and it will be exceptionally difficult to adopt such strategies to the next generation of simulations.

This paper proposes a new approach that allows extraction of physically meaningful and temporally coherent features from unsteady flows, one time-step at a time, by computing appropriate reference frames. Note that both the indicatorbased and the pathline-based approaches implicitly aim to process the data in a more useful reference frame, yet neither technique tries to construct such a frame. Instead, we propose to revisit the earlier argument [Lug79,PT84,PC94] and explicitly compute a more suitable reference frame. More specifically, we introduce the notion of an internal reference frame. The internal frames can be computed in an efficient and massively-parallel manner, and are more general than uniformly moving frames, as their motion is represented by a harmonic flow. Using several case studies, we show that the internal frames are indeed distinguished, and are able to extract important coherent features in large turbulent flows. An important consequence of using the proposed frames is that the large body of latent research on steady flow analysis becomes relevant for unsteady flows, with the potential for significant scientific impact. In particular, we:

1. Define the internal frame as the one whose motion is described by the natural harmonic flow [BPB14];

2. Propose a new efficient, parallel, and scalable algorithm to compute the natural harmonic flow using only the boundary data, which leads to the internal frame for the given flow defined on a simply-connected subset of $\mathbb{R}^{n}$;

3. Discuss how a localized analysis can be performed using internal frames for a more targeted analysis on smaller regions of interest; and

4. Analyze several large-scale flows, and demonstrate that the proposed internal frame is a distinguished frame that enables extraction of meaningful and coherent features.

\section{Related Work}

The analysis of unsteady flow has been an important topic of research in the fluids and visualization communities. A detailed survey on this subject was published by Pobitzer et al. [PPF* 10]. Perry and Tan [PT84] pointed out a key insight in this context that meaningful analysis of unsteady flow requires a reference frame where the flow becomes steady or near-steady, and proposed to extract features from ocean flows in a frame that is moving with eddies. They suggested that there may not exist a single reference frame that allows extraction of all important features. However, assuming linearly moving critical points, Perry and Chong [PC94] stressed the importance of Galilean invariance, stating that using a uniformly moving frame is sufficient in most cases.

Based largely on these arguments, a large number of techniques have been developed focusing on obtaining Galilean invariance. In particular, the scalar indicators to detect vortices, such as the Q- [Hun87], $\Delta-$ [CPC90], and $\lambda_{2}-$ [JH95] criteria are all Galilean invariant, since they are derived from the deformation tensor, which, as a gradient, ignores constant motion. Other properties of the deformation tensor have also been used to classify regions with specific flow behavior [CPC90, CPL $\left.{ }^{*} 11\right]$. Kasten et al. [KRHH11] and Sahner et al. [SWH05] also extract vortex cores based on Galilean-invariant quantities - acceleration magnitude and vortex indicators such as $\lambda_{2}$, respectively.

However, Galilean invariance does not suffice for all cases, especially when a non-uniformly moving object is involved. Haller [Hal05] proposed an "objective" definition of vortex, which is invariant to more general motions of frames, namely, time-dependent translations and time-dependent rotations. Acknowledging the need for accelerating frames, 
Fuchs et al. [FKS*10] proposed to use an "unsteadiness" metric based on the material derivative of the Jacobian, whose minima was then used to extract coherent features. There also exist examples, such as the double gyre [SLM05] and the petri dish $\left[\mathrm{WCW}^{*} 11\right]$, where there does not exist a known simple frame that would reveal the features of interest. However, a large number of use cases in areas such as industrial design of automobiles, aeroplanes, ships, turbines, etc., as well as science applications such as climate or combustion could likely benefit from proper reference frames as demonstrated by our results.

Instead of trying to find new reference frames, the visualization and analysis community has shifted its focus to Lagrangian or particle-based techniques. These approaches trace pathlines in the flow to compute the FTLE [Hal00], which is then used to highlight the Lagrangian coherent structures (LCS) [Hal01] that represent flow barriers. However, as discussed in Section 1, such techniques have severe limitations such as high computational cost, dependence upon the "correct" time-scale for analysis, and dependence upon the availability of a large number of time-steps.

\section{Vector Field Decomposition}

Computing internal reference frames is intimately connected with vector field decompositions. This section discusses the mathematical background for some relevant decompositions before defining the internal frame in Section 4.

\subsection{Localized Flow}

Wiebel et al. [WGS07] propose to decompose a flow into two components - potential and localized. As the name suggests, the localized flow captures the local properties of the flow by representing its divergence and rotation inside the domain, whereas the potential flow has no divergence or rotation, and matches the original flow at the boundary of the domain. Removing the potential flow from a given flow is equivalent to creating a new reference frame that allows extraction of local and formerly-obscured features.

The most important limitation of this approach is that the localized flow is always confined within the domain, i.e., its component normal to the boundary is zero. This is a strong assumption, especially for compressible flows. For example, consider a nodal source in a closed domain: One would expect the localized flow corresponding to the source to cross the domain boundary yet this violates the prior assumption. Furthermore, the technique needs additional information (material density) to resolve compressible flows. Finally, as mentioned by the authors [WGS07], the material density should be near constant for the technique to apply to unsteady flows. At large, however, this decomposition can be seen as an incomplete variant of a more general flow decomposition called the Helmholtz-Hodge decomposition.

\subsection{The Helmholtz-Hodge Decomposition (HHD)}

The Helmholtz-Hodge decomposition (HHD) [Hel58] decomposes a vector field into irrotational, incompressible, and harmonic vector fields. Consider a smooth vector field $\vec{V}: \Omega \rightarrow \mathbb{R}^{n}$, where $\Omega \subseteq \mathbb{R}^{n}$ (for $n=2,3$ ), then

$$
\vec{V}=\vec{d}+\vec{r}+\vec{h},
$$

where $\vec{d}$ is irrotational $(\nabla \times \vec{d}=\overrightarrow{0}), \vec{r}$ is incompressible $(\nabla \cdot \vec{r}=0)$, and $\vec{h}$ is harmonic $(\nabla \times \vec{h}=\overrightarrow{0}$ and $\nabla \cdot \vec{h}=0)$. This leads to the following equalities:

$$
\nabla \cdot \vec{d}=\nabla \cdot \vec{V}, \quad \text { and } \quad \nabla \times \vec{r}=\nabla \times \vec{V} .
$$

Since a harmonic flow is both irrotational and incompressible, it can be represented as any of the components $\vec{d}, \vec{r}$, or $\vec{h}$ in (1), thus adding a degree of freedom. Therefore, in cases where a harmonic flow can exist, i.e., for domains with boundary, or for non-simply connected domains, the decomposition is not unique. The traditional way to obtain a unique solution is to specify a set of boundary conditions.

To compute the decomposition, the components $\vec{d}$ and $\vec{r}$ are represented as the gradient of a scalar potential $D$, and the curl of a vector potential $\vec{R}$, respectively. Substituting $\vec{d}=\nabla D$ and $\vec{r}=\nabla \times \vec{R}$ in (2), we get two Poisson equations,

$$
\Delta D=\nabla \cdot \vec{V}, \quad \text { and } \quad \vec{\Delta} \vec{R}=-\nabla \times \vec{V},
$$

where $\Delta$ is the (scalar) Laplacian, i.e., $\Delta=\nabla^{2}$, and $\vec{\Delta}$ is the vector Laplacian, i.e., $\vec{\Delta} \vec{R}=\nabla(\nabla \cdot \vec{R})-\nabla \times(\nabla \times \vec{R})$, with $\nabla \cdot \vec{r}=0$ implies $\nabla \cdot \vec{R}=0$ due to the gauge condition [Gir88]. Assuming some boundary conditions, the Poisson equations (3) are solved uniquely, leading to the components $\vec{d}$ and $\vec{r}$. Subsequently, the harmonic component is computed as the remainder: $\vec{h}=\vec{V}-\vec{d}-\vec{r}$.

In $2 \mathrm{D}$, curl can be represented as a scalar value in the normal direction to the domain. This leads to a simpler representation of $\vec{r}$ as the co-gradient of a scalar potential $R$, i.e., $\vec{r}=J \nabla R$, where $J$ is defined as the $\pi / 2$-rotation operator.

\subsection{Longitudinal and Transverse Components}

A particularly important 2-component form of the HHD is

$$
\vec{V}=\nabla D+\nabla \times \vec{R},
$$

where, the component $\nabla D$ is called the longitudinal and $\nabla \times \vec{R}$ the transverse component of the flow [Arf85, Ste11]. The corresponding potentials $D$ and $\vec{R}$ are defined as

$$
\begin{aligned}
D\left(\mathbf{x}_{0}\right)=\int_{\Omega} G_{\infty}\left(\mathbf{x}, \mathbf{x}_{0}\right) & (\nabla \cdot \vec{V}(\mathbf{x})) \mathrm{d} \mathbf{x} \\
& -\oint_{\partial \Omega} G_{\infty}\left(\mathbf{x}, \mathbf{x}_{0}\right)(\vec{n} \cdot \vec{V}(\mathbf{x})) \mathrm{d} \mathbf{x}, \\
\vec{R}\left(\mathbf{x}_{0}\right)=-\int_{\Omega} G_{\infty}\left(\mathbf{x}, \mathbf{x}_{0}\right)(\nabla \times \vec{V}(\mathbf{x})) \mathrm{d} \mathbf{x} & \\
& +\oint_{\partial \Omega} G_{\infty}\left(\mathbf{x}, \mathbf{x}_{0}\right)(\vec{n} \times \vec{V}(\mathbf{x})) \mathrm{d} \mathbf{x},
\end{aligned}
$$


where $\vec{n}$ is the outward normal to the boundary, and $G_{\infty}\left(\mathbf{x}, \mathbf{x}_{0}\right)$ is the free-space Green's function, such that

$$
\begin{aligned}
G_{\infty}\left(\mathbf{x}, \mathbf{x}_{0}\right) & =\frac{1}{2 \pi} \log \left(\left|\mathbf{x}-\mathbf{x}_{0}\right|\right) & \mathbf{x}, \mathbf{x}_{0} \in \mathbb{R}^{2}, \\
G_{\infty}\left(\mathbf{x}, \mathbf{x}_{0}\right) & =\frac{-1}{4 \pi\left|\mathbf{x}-\mathbf{x}_{0}\right|} & \mathbf{x}, \mathbf{x}_{0} \in \mathbb{R}^{3} .
\end{aligned}
$$

This decomposition is defined only for simply connected subsets of $\mathbb{R}^{n}$, and distributes any harmonic component between $\nabla D$ and $\nabla \times \vec{R}$.

\section{The Internal Reference Frame}

A recent survey [BNPB13] discusses a large number of applications that use the HHD to extract vector field features, most of which, however, ignore the well-known boundary artifacts common in the traditional decomposition. Recently, Bhatia et al. [BPB14] showed that these artifacts are caused by incompatible boundary conditions, which effectively add a non-zero harmonic flow to the incompressible and irrotational components. They also point out that a harmonic flow on a given domain is always independent of the divergence and rotation present inside the domain, and is, in fact, caused by factors external to the domain. They propose a new variant of the HHD, called the natural HHD, which guarantees that the incompressible and irrotational components are influenced only by the properties inside the domain. Hence, no boundary conditions are required for a unique HHD, and boundary artifacts are avoided.

\subsection{Understanding the Internal Reference Frame}

An important consequence of the natural HHD is that, by definition, the natural harmonic flow, $\vec{h}^{*}$, captures all external influences on the flow [BPB14]. This paper proposes that it is this property that makes $\vec{h}^{*}$ an excellent choice to describe a meaningful reference frame. Essentially, using the natural HHD, the motion of each particle in the flow can be expressed as a combination of two components: caused due to (1) internal and (2) external influences. Thus, creating new particles, whose motion is described by $\vec{h}^{*}$, is equivalent to defining new observers that compensate for all external influences. In this new "reference frame," the internal properties of the flow can be observed directly, and we therefore call it the internal reference frame.

Definition 4.1 (Internal Reference Frame) A flow field is said to be analyzed in the internal reference frame if it is invariant to external influences (harmonic flow).

We point out that we use the term "reference frame" in a physical sense to characterize the state of motion of an observer, as done by Lugt [Lug79], also referred to as observational frame in the Newtonian relativity [KB03], where physical quantities are measured with respect to the state of motion of an observer. It should not be confused with the mathematical notion of a frame of reference that denotes a coordinate system. To understand the significance of the internal frames, consider a passenger walking inside a moving train. A person standing outside the train represents a static reference frame, and cannot find out the speed of the walking passenger without knowing the train's speed. However, another passenger sitting inside the train can accurately describe the walking passenger's movements since he represents a moving frame that already compensates for the train's motion. In the context of flow fields, the walking passenger represents the internal properties of the flow, the outside observer represents the frame in which the flow is simulated, and the second passenger the internal frame. For a uniformly moving train, the reference frame is trivial, as each passenger experiences the same influence from the train. However, for more general motions, e.g., a train on a curved track, each passenger will experience different influence depending upon its position within the train, and the radius and the center of the curve. As a result, each passenger (flow particle) must define a separate internal frame only for itself.

\subsection{Transforming the Flow into the Internal Frame}

In order to transform the data into the internal frame, we make use of the definitions provided by the natural HHD [BPB14]. The natural irrotational $\left(\vec{d}^{*}=\nabla D^{*}\right)$ and the natural incompressible $\left(\vec{r}^{*}=\nabla \times \vec{R}^{*}\right)$ components of the flow $\vec{V}$ are defined using the natural potentials $D^{*}$ and $\vec{R}^{*}$.

$$
\begin{aligned}
& D^{*}\left(\mathbf{x}_{0}\right)=\int_{\Omega} G_{\infty}\left(\mathbf{x}, \mathbf{x}_{0}\right)(\nabla \cdot \vec{V}(\mathbf{x})) \mathrm{d} \mathbf{x}, \\
& \vec{R}^{*}\left(\mathbf{x}_{0}\right)=-\int_{\Omega} G_{\infty}\left(\mathbf{x}, \mathbf{x}_{0}\right)(\nabla \times \vec{V}(\mathbf{x})) \mathrm{d} \mathbf{x} .
\end{aligned}
$$

Note that the computation of these components require only the divergence and rotation of $\vec{V}$ inside the domain. Since no boundary conditions are required to compute $D^{*}$ and $\vec{R}^{*}$, these potentials contain no harmonic flow and hence no external influences. Using (6), we can rewrite (4) as

$$
\begin{aligned}
& D\left(\mathbf{x}_{0}\right)=D^{*}\left(\mathbf{x}_{0}\right)-\oint_{\partial \Omega} G_{\infty}\left(\mathbf{x}, \mathbf{x}_{0}\right)(\vec{n} \cdot \vec{V}(\mathbf{x})) \mathrm{d} \mathbf{x}, \\
& \vec{R}\left(\mathbf{x}_{0}\right)=\vec{R}^{*}\left(\mathbf{x}_{0}\right)+\oint_{\partial \Omega} G_{\infty}\left(\mathbf{x}, \mathbf{x}_{0}\right)(\vec{n} \times \vec{V}(\mathbf{x})) \mathrm{d} \mathbf{x} .
\end{aligned}
$$

Equating the natural HHD with the longitudinal and transverse decomp., $\nabla D^{*}+\nabla \times \vec{R}^{*}+\vec{h}^{*}=\nabla D+\nabla \times \vec{R}$, we get

$$
\begin{aligned}
\vec{h}^{*} & =\nabla\left(D-D^{*}\right)+\nabla \times\left(\vec{R}-\vec{R}^{*}\right), \\
\Longrightarrow \vec{h}^{*} & =\nabla H_{d}^{*}+\nabla \times \vec{H}_{r}^{*},
\end{aligned}
$$

where

$$
\begin{aligned}
& H_{d}^{*}\left(\mathbf{x}_{0}\right)=-\oint_{\partial \Omega} G_{\infty}\left(\mathbf{x}, \mathbf{x}_{0}\right)(\vec{n} \cdot \vec{V}(\mathbf{x})) \mathrm{d} \mathbf{x}, \\
& \vec{H}_{r}^{*}\left(\mathbf{x}_{0}\right)=\oint_{\partial \Omega} G_{\infty}\left(\mathbf{x}, \mathbf{x}_{0}\right)(\vec{n} \times \vec{V}(\mathbf{x})) \mathrm{d} \mathbf{x} .
\end{aligned}
$$

Thus, the natural harmonic flow, which represents all external influences, can be computed using the two boundary integrals of (8). 
To represent the internal frame, we create particles that move along the natural harmonic flow, $\vec{h}^{*}$. By the velocity addition formula of Newtonian relativity, such particles observe the original flow as $\vec{V}_{\text {int }}=\vec{V}-\vec{h}^{*}$. In other words, the flow field $\vec{V}$ can be directly transformed into the internal frame by removing the natural harmonic component. For unsteady flows $\vec{V}(\mathbf{x}, t)$, this transformation can be applied to each time-step, leading to smooth $\vec{V}_{\text {int }}(\mathbf{x}, t)$ that can be used for extraction of meaningful features, as demonstrated later.

Internal Frame and Topology of the Domain. It is important to note that (7) is valid only for simply connected subsets of $\mathbb{R}^{n}$. For non-simply connected domains, there may exist other forms of harmonic flow induced by the topology of the domain, as explained by the Hodge-Morrey-Friedrichs decomposition [Sch95]. As a result, it is not guaranteed that all harmonic flow can be represented by (7). However, this is only a conceptual limitation and does not restrict the practical utility of the proposed technique for three reasons: (i) Most simulations impose no-slip boundary conditions at fluid-solid interfaces, i.e., $\vec{V}=0$. In such cases, a smooth $\vec{V}_{0}$ can be defined on a simply connected $\Omega_{0}(\supset \Omega)$ by padding $\vec{V}$ with zeros in the holes of $\Omega$. It can be shown that this padding does not influence the internal frame. Therefore, the internal frame can be computed easily for $\vec{V}_{0}$ on $\Omega_{0}$ but used to analyze $\vec{V}$ on $\Omega$. (ii) Furthermore, many practical flows, such as the flow behind a cylinder (see Section 5.1) do not contain any additional forms of harmonic, and therefore (7) is applicable. (iii) Finally, even when cases (i) and (ii) are not applicable, the flow in the internal frame can be computed indirectly using the natural HHD, i.e., $\vec{V}_{\text {int }}=\vec{d}^{*}+\vec{r}^{*}$.

Localized Internal Frame. Since, the internal frame is defined on a point-wise basis and depends only on the boundary data, it can be computed with respect to an arbitrary shaped boundary, as long as it bounds a simply connected subset of $\mathbb{R}^{n}$. This allows computing a localized internal frame for a more focused analysis in a smaller region of interest and to highlight local features. Furthermore, this technique also allows for a local computation, meaning that one can define the frame for a large domain, but compute it only in a smaller subset. This further helps reducing the computational costs by focusing only on the regions of interest. Recall that $\vec{V}_{\text {int }}$ depends only upon the divergence and rotation of $\vec{V}$ inside the domain considered. Therefore, from the smoothness of $\vec{V}$, it follows that $\vec{V}_{\text {int }}$ will vary smoothly with respect to smooth changes in the domain considered.

\subsection{Implementation}

Computation of the flow in the internal frame requires two modules: (i) computation of divergence, curl, and gradient; and (ii) computation of boundary integrals of normal and tangential components of the flow. With the availability of these modules, its computation using (8) is straightforward and trivially parallelizable for a variety of spatial discretizations and/or interpolants. For brevity, we only discuss bilinear/trilinear flows defined on regular grids, and piecewise constant (PC) flows defined on triangulated meshes.

For a regular grid $\mathcal{G}$ with vector samples defined on vertices, the normal and tangential components of the boundary flow can be defined trivially with respect to the exterior normal, once an orientation is assumed with respect to Cartesian coordinates. Since each "side" of $\mathcal{G}$ is also a regular grid, integration along the boundary can be approximated using trapezoidal method applied successively in each dimension. Finally, central finite differences can be used to define the required differential operators.

For a triangulated domain $\mathcal{T}$ with a PC vector field defined on its faces, the normal and tangential components are computed with respect to the edges on the boundary, and boundary integrals are approximated by summing these components. The divergence (and curl) of the vector field is defined at the vertices of $\mathcal{T}$, and is computed as the sum of dot products of the vector field with the normal (and tangent) along the boundary of the one-ring neighborhood of the vertex [PP03]. The gradient of a scalar field defined at vertices of $\mathcal{T}$ is a vector field defined at its faces. For each face, it is computed as the sum of the gradients of its barycentric coordinates weighted by the corresponding function values.

\section{Results}

We show the results of our analysis on simulated flows representing physical phenomena of interest. First, we use the flow behind a cylinder to compare different frames of reference. Since the resulting features of this flow are well known, we use this data to demonstrate that the internal frame extracts a vortex street whose behavior is consistent with theory, and is indeed a distinguished frame. Next, we use the internal frame to explore complex flows resulting from combustion simulations, and show that we extract features otherwise non-trivial to observe.

To show that instantaneous techniques are applicable in the internal frame, we compute the critical points and the saddle separatrices of the flow. These features define the topological skeleton of the flow, which is used to decompose the domain into non-overlapping regions. Most of the following figures show this topological decomposition by highlighting these regions in different colors overlayed on the image-based flow visualization (IBFV) [vW02]. In the remaining figures, the IBFV is overlayed with the color-map shown here to represent vector magnitudes, with blue-to-red representing low-to-high values. Blue spheres denote saddles in the flow, while red and green spheres denote clockwise and counter-clockwise rotating vortices. All performance numbers were noted for double precision data and serial algorithm running on a MacBook Pro with 2.8 GHz Intel Core 2 Duo processor and 8 GB RAM. 


\subsection{Flow behind a cylinder}

We first study the 2D flow behind a cylinder, which is expected to exhibit the well known von Kármán vortex street, as illustrated in Figure 2.

Using the data for Reynolds number, $R e=165$ and inlet velocity, $u=1.1$, it is observed in Figure 3 that the topological decomposition of the flow in the simulation's reference frame is trivial, since the entire flow originates from the left boundary and exits from the right. However, the same analysis performed in the internal frame reveals the expected vortex street. This is a well studied data, typically used for illustrating vortex detection techniques, most of which rely on scalar indicators associated with thresholds [KRHH11]. The internal frame allows the computation of such regions by analyzing the flow directly from a single time-step, while maintaining all information about it. For example, the internal frame also reveals the region of backward flow (highlighted by arrows) between the counter-rotating vortices, which induces drag on the cylinder, whereas the original frame obscures this behavior. Furthermore, neither the techniques based on scalar indicators nor the FTLE-based approaches are able to represent backward flow due to information loss during the corresponding transformations.

To prove that the internal reference frame is indeed a distinguished frame for analysis, we show in Figure 4 that analysis in slightly slower or faster frames produces results inconsistent with theory. In particular, the vortex street is expected to be vertically symmetric (with horizontal offsets) about the center of the cylinder at all times because both the cylinder and the inlet velocity are vertically symmetrical. However, for slower and faster frames, the instantaneous flows are observed to be moving, more or less, downward and upward respectively. Notice also that the amount of background flow exhibited in the slower frame is significantly reduced, while the faster frame exhibits inverse flow at the top of the domain.

Next, we analyze the flows for increasing Reynolds numbers, $R e=100,125,140$, and 165, corresponding to a fixed kinematic viscosity and $u=0.92,0.98,1.01$, and 1.1 . To verify the temporal coherence of the results, we compute the vortices for the simulation time-range [10,22.5] consisting of 26 snapshots, one at a time. Figure 5 shows the trace of some of the vortices extracted from these snapshots using one spatial domain to encode time. As expected, the locations of the critical points track the inherent motion of the vortices allowing accurate measurement of the "speed" of the vortex cores. Our results confirm that the vortices move slightly slower than $(\approx 91 \%$ of $)$ the inlet velocity. In the shown example, the speed of vortices are $\approx 0.85, \approx 0.89$, $\approx 0.92$, and $\approx 1$. Note that flows in the internal frame exhibit temporal stability, and so no actual tracking of the vortices was necessary to produce this results; instead the figures simply show the position of the vortex core at the given time steps. We also note that an approximation to shedding

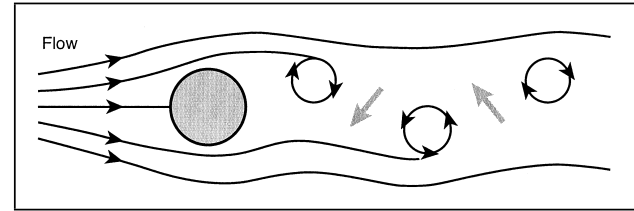

Figure 2: Illustration of the expected von Kármán vortex street [DL02]. Notice the backward flow between counterrotating vortices that induces drag on the cylinder.

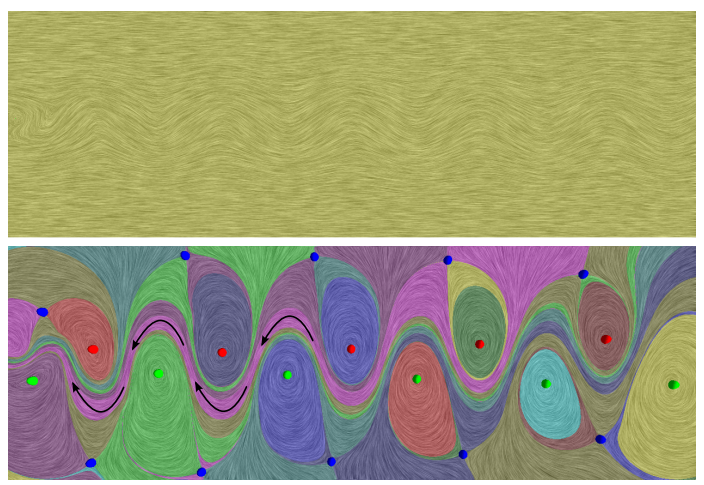

Figure 3: Topological decomposition of the flow behind a cylinder is trivial in the original frame (top), while the same analysis in the internal frame (bottom) reveals the vortices, with backward flow between them. The internal frame for this $[1450 \times 400]$ data took $\approx 43$ sec. to compute.

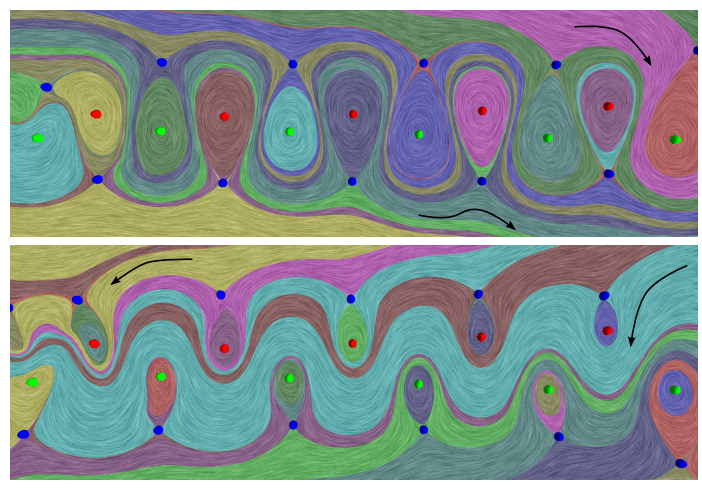

Figure 4: Slowing down (top) or speeding up (bottom) the internal frame yields inconsistent results. The shown results for $10 \%$ changes to the internal frame clearly demonstrate that the flow is no longer vertically symmetrical.

frequency computed using our technique increases with increasing Reynolds number - a trend consistent with theory.

Finally, we compare our topological analysis in the internal frame, with the particle-based and indicator-based techniques, both in the simulation's frame. Figure 1 shows a few pathlines computed in the simulation's frame, along with IBFV of the flow in the internal frame. On the left and the right, we zoom-in to show the position of these particles at $t=10$ and $t=22.5$, overlayed on the topological decomposition computed in the internal frame, and the contours 
H. Bhatia et al. / Extracting Features from Time-Dependent Vector Fields Using Internal Reference Frames
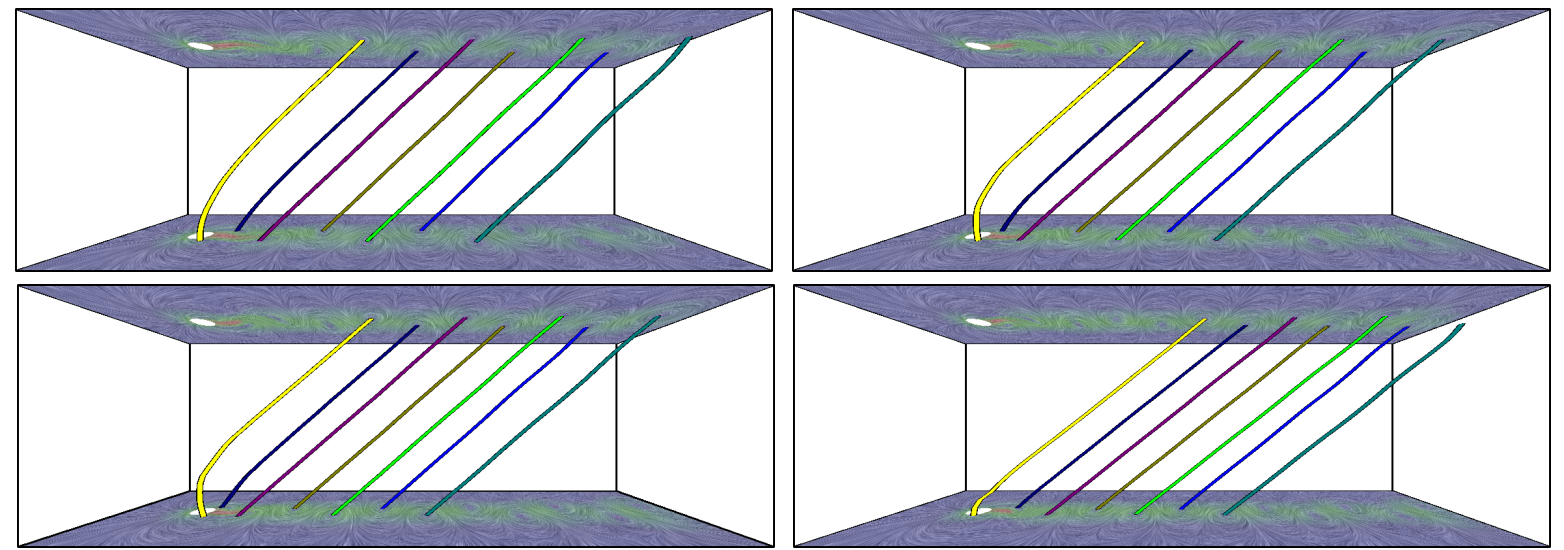

Figure 5: Vortex traces in the flow behind the cylinder. From top-left to bottom-right, with increasing Re, the speed of vortices increases as expected. To reduce clutter, only those vortex traces are shown that exist for the entire time range.

of Q-criteria [Hun87] computed in the simulation's frame. The result asserts that the vortex identification using $\mathrm{Q}$ can create false-positives and false-negatives, e.g., the olive and the green colored particles, respectively. Notice that the olive pathline does not swirl around the vortex core, while the green pathline does. In contrast, the topologically identified vortex in the internal frame captures the true swirling behavior of the particles, from a single time-step and at much lower computational expense.

\subsection{Lifted Ethylene Jet Flame}

Next, we study the direct numerical simulation of a turbulent lifted ethylene jet flame [YRSC11], which represents a compressible and highly turbulent flow. In this case, fuel is injected from the bottom of the domain creating a strong background flow towards the top. The natural harmonic flow, which represents the motion of the internal frame, reveals an elliptical shape (see Figure 6(b)) consistent with the nonuniform velocity profile (faster flow in the middle) imposed by the simulation. This is an example of non-uniformly moving frames that cannot be compensated for using Galileaninvariant techniques. In contrast, our technique successfully decouples this frame.

As shown in Figure 6(c), analyzing a single snapshot of the flow in internal reference frame reveals two global counter-rotating vortices rather than a streak of smaller vortices one may have expected. Instead, the smaller vortices are nested in these global rotating structures. The figure also highlights the flow that runs through the domain from bottom to top, along with all the smaller structures that exist on either side of this through-flow. As is clear from the figure, none of these intrinsic features are visible in the simulation's reference frame (see Figure 6(a)).

Figure 7 shows traces of vortices for time $[0.00174,0.00175]$, confirming that the vortices are temporally coherent. While many vortices are small and

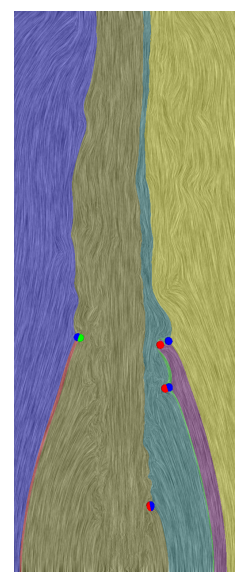

(a)

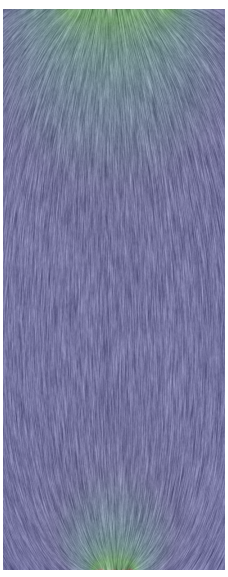

(b)

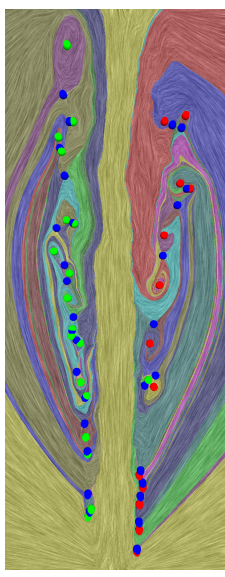

(c)
Figure 6: Comparison of the topological decomposition of the lifted ethylene jet flame in the simulation's (left) and the internal (right) reference frames. The natural harmonic flow (middle) describes the motion of the internal frame. Only 8 critical points are identified in the original frame, while 62 critical points describe the complex dynamics of this simulated flow in the internal frame. The internal frame for this $[800 \times 2025]$ data took $\approx 186 \mathrm{sec}$. to compute.

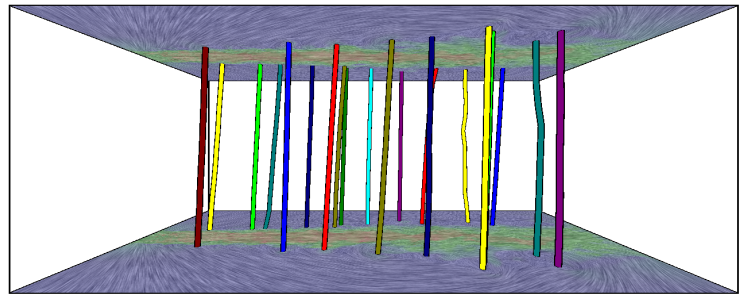

Figure 7: The traces of vortices show the presence of stable rotational structures in the ilfted ethylene flame.

cancel out with saddles in the simulation, some that are stable and persist for the entire time-range are shown. 


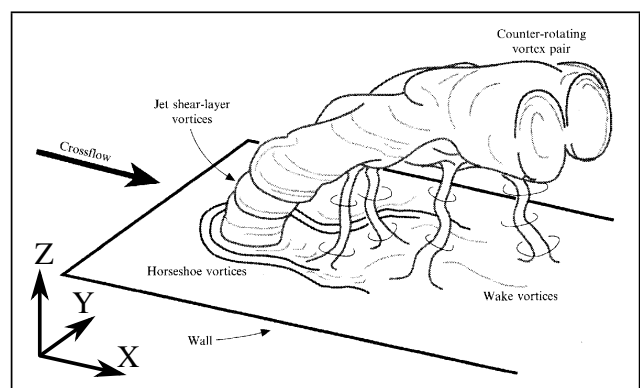

Figure 8: Illustration [FR94] of the four types of vortical structures associated with this flow: jet shear-layer vortices at the perimeter of the bending jet, the developing counterrotating vortex pair, horseshoe vortices on the wall, and wake vortices extending from the wall to the jet. The orthogonal axes assumed for analysis are shown, and the dimensions of the dataset are $[1408 \times 1080 \times 1100]$.

\subsection{Jet in Cross-Flow}

The next data represents a jet in cross-flow [GGK*12], which is a fundamental flow phenomenon relevant to many engineering applications, e.g., film cooling of turbines, fuel injections, and dilution jets in gas-turbine combustors. The experimental set-up contains injection of flow through a jet at the bottom in the presence of a strong background flow in transverse direction, the cross-flow, as illustrated in Figure 8. The goal of the experiment is to study different types of vortical structures created by the interaction of the burning jet with the cross-flow. The most prominent structures in this flow are a pair of counter-rotating vortices, which occur as a result of the impulse of the jet on the cross-flow, and become dominant in the far field. At the periphery of the jet's inflow, jet shear-layer vortices are created due to the annular shear layer that separates from the edge of the jet orifice. These vortices dominate the initial portion of the jet. The horseshoe vortices wrap around the base of the jet issuing from a wall into a cross-flow, and the wake vortices are the structures existing between the bottom wall and the jet itself.

We show results obtained in the internal frame for selected $x z$ and $y x$ slices. Since the cross flow is transversal to $y z$ planes, the simulation's frame is already very similar to the internal frame. First, we analyze a $x z$-plane for $y=550$, which cuts through the center of the jet. Figure 9 shows the topological decomposition of the flow in the original and the internal frames. Notice the vortical structures revealed in the internal frame. To emphasize the differences, we show the zoomed-in region surrounding the jet, and notice the presence of shear-layer vortices around the jet. Other vortices are also observed, which are not revealed in the original frame.

Next, we study the topological decomposition of the flow in the internal frame for slices representing $y x$-planes. As shown in Figure 10, interesting features are observed for slices $z=50$ and $z=350$, both of which cut through the jet transversally. The first slice is near the orifice, and shows the formation of two counter-rotating vortices in a nascent stage (highlighted as rotation). This helps in observing how the size of these vortices grows with increasing $z$. Other smaller vortices are observed above these structures, which appear to be the wake vortices (highlighted using arrows). As expected at a greater height $(z=350)$, one observes these vortices to become stretched in the $x$-direction (vertical in the figure) as the plane cuts through a greater part of the vortices, while the wake vortices now appear below them.

Localized 3D computation. Finally, we present results on the $3 \mathrm{D}$ flow in the internal frame. Chosen by scientists, we analyze a $[400 \times 200 \times 250]$ subset covering the most interesting region of the flow. Figure 11(a) shows the natural harmonic flow computed for this region. Figures 11(b) and 11(c) show two views of the flow in the internal frame, highlighting different structures. The first view focuses at the jet, and shows the vortices created around it, while the second shows the formation of the counter-rotating vortex pair, a structure notoriously difficult to extract. The challenge is that the vortical motion is significantly weaker than that of the smaller structures in the interior, which therefore, dominate traditional detection techniques. In fact, several of our scientific collaborators have remarked that this is the first time they have seen this structure visualized directly.

\section{Discussion and Outlook}

This paper promotes the idea of computing new reference frames that allow extraction of coherent features from individual time-steps. We believe that the success of the proposed internal frames will revive the abandoned idea of analyzing unsteady flows one time-step at a time. Our results demonstrate that the analysis in the internal frame captures the dynamics of material particles, and extracts temporally coherent features. While there are examples where the existence of a distinguished frame is not yet known, e.g., the double gyre [SLM05] and the petri dish [ $\left.\mathrm{WCW}^{*} 11\right]$, the proposed frames address a large class of flows that represent physical phenomena in the presence of a background flow or around a moving object. In particular, the motion of the internal frames is represented by a (possibly time-varying) harmonic flow, thus capturing more general background flows than uniformly moving.

We hope that the proposed work will foster new research towards computing more general frames, e.g., rotational, that can help analyze other types of flows as well. The proposed ideas make a large number of existing techniques for steady flow analysis directly applicable to unsteady flows. Consequently, the proposed work can open new horizons in in-situ analysis of large-scale complex flows, where simulations are temporarily halted to analyze the current state and only the results are dumped. Especially with anticipated advances in compute power and lingering I/O bottlenecks, such analysis can enhance our ability to analyze data of ever-growing 
H. Bhatia et al. / Extracting Features from Time-Dependent Vector Fields Using Internal Reference Frames

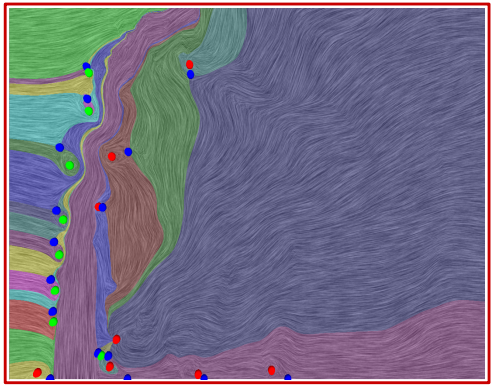

(a)

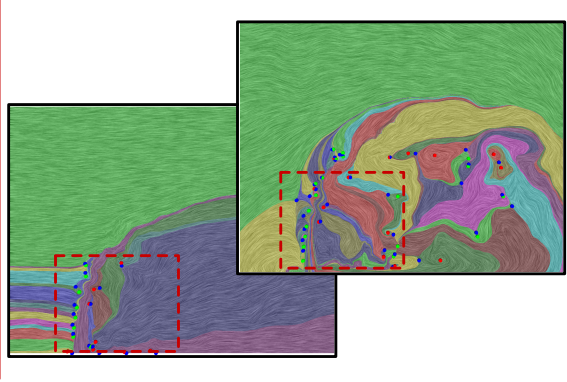

(b)

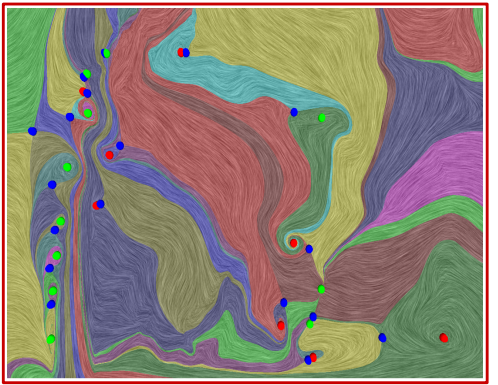

(c)

Figure 9: Topological decomposition of the jet in a cross-flow (b bottom, a) shows little structure as the interesting intrinsic phenomena are overshadowed by the background flow. The same topological analysis to the flow in the internal frame ( $b$ top, c) reveals the expected shear-layer vortices on the front of the flame as well as a wealth of other features.
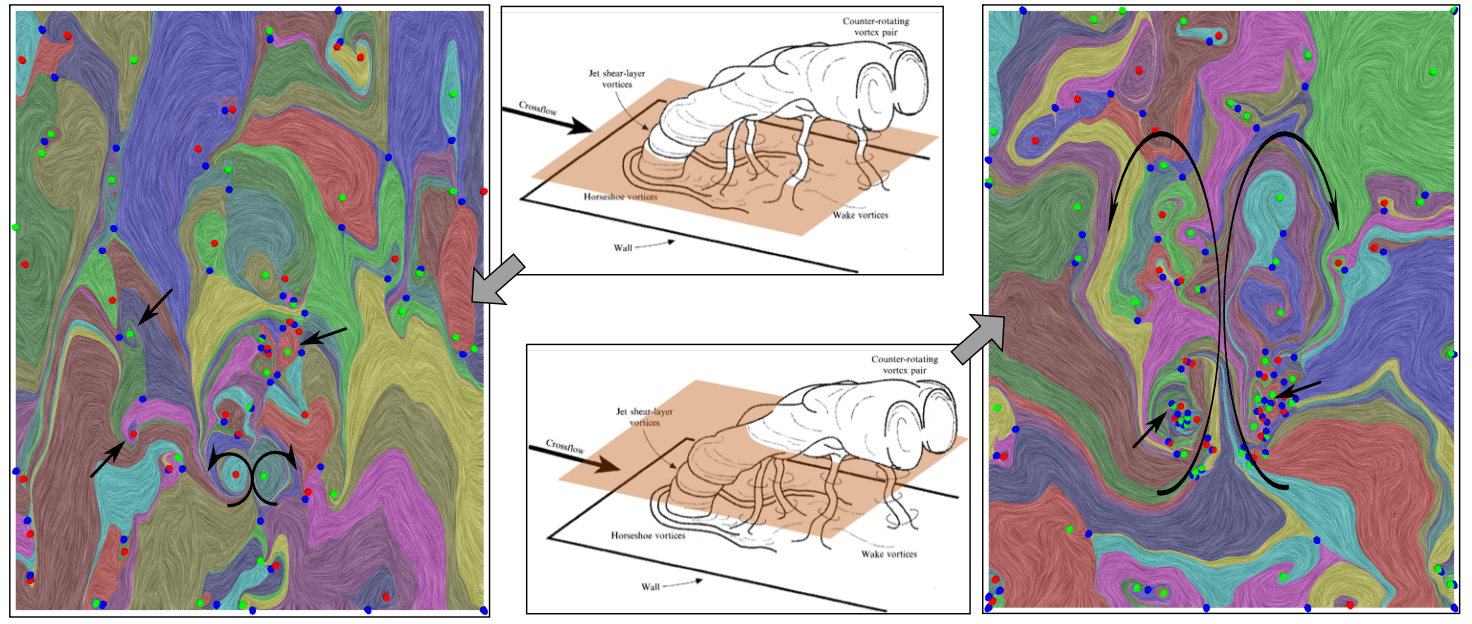

Figure 10: Topological decomposition of the flow in the internal frame for the yx slices shows the interior of the jet near its orifice, and the evolution of jet with height. Observed wake vortices are marked with arrows.

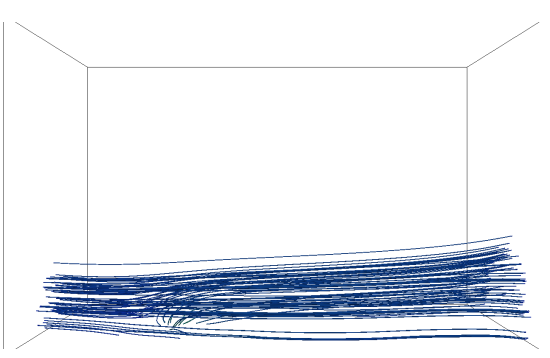

(a)

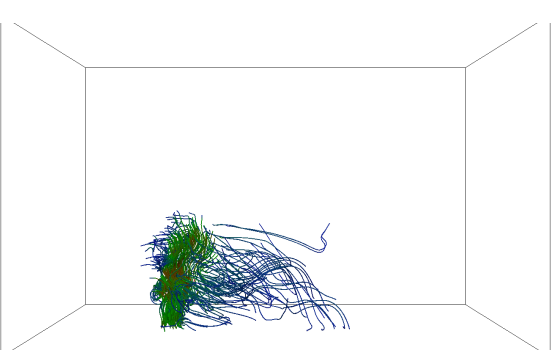

(b)

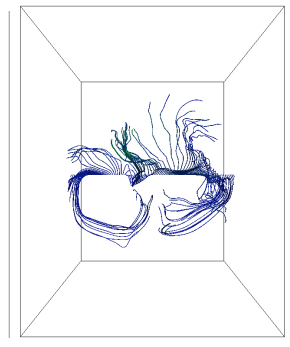

(c)

Figure 11: Localized analysis on a 3D subset of the flow. (a) The natural harmonic flow. $(b)$ and (c) Flow in the internal frame highlighting features near the jet orifice $(b)$ and the formation of counter-rotating vortex pairs (c).

scale and complexity at much higher temporal resolutions than otherwise.

\section{Acknowledgements}

We are thankful to Mahsa Mirzargar from the SCI Institute for simulating the flow behind the cylinder, used in
Section 5.1, using Nektar++ [Nek13], and Jacqueline Chen from the Sandia National Laboratory for the flows used in Sections 5.2 and 5.3. This work was performed under the auspices of the US Department of Energy (DOE) by Lawrence Livermore National Laboratory (LLNL) under contract DE-AC52-07NA27344. LLNL-CONF-650513. 


\section{References}

[Arf85] ARfKen G.: Mathematical Methods for Physicists, 3rd ed. Academic, New York, 1985. 3

[BMP06] Braun S. A., Montgomery M. T., Pu Z.: Highresolution simulation of hurricane bonnie (1998). Part I. Journal of the Atmospheric Sciences 63, 1 (2006), 19-42. 1

[BNPB13] Bhatia H., Norgard G., PASCUCCi V., BREMER P.-T.: The Helmholtz-Hodge decomposition - A survey. IEEE Trans. on Vis. and Comp. Graph. 19, 8 (2013), 1386-1404. 4

[BPB14] Bhatia H., PASCUCCI V., BREMER P.-T.: The natural Helmholtz-Hodge decomposition for open-boundary flow analysis. IEEE Trans. on Vis. and Comp. Graph. (2014). To Appear. 2,4

[CPC90] Chong M. S., Perry A. E., Cantwell B. J.: A general classification of three-dimensional flow fields. Physics of Fluids A 2, 5 (1990), 765-777. 2

[CPL*11] Chen G., Palke D., Lin Z., Yeh H., Vincent P., LARAMEE R. S., ZHANG E.: Asymmetric tensor field visualization for surfaces. IEEE Trans. on Vis. and Comp. Graph. 17, 12 (2011), 1979-1988. 2

[DL02] DRUCKER E. G., LAuder G. V.: Experimental hydrodynamics of fish locomotion: Functional insights from wake visualization. Integr. and Comp. Biol. 42 (2002), 243-257. 6

[FKS*10] FuChS R., KeMmLER J., SCHINDLER B., WASER J., SAdlo F., HAUSER H., PEIKERT R.: Toward a Lagrangian vector field topology. Comp. Graph. Forum (Proc. of EuroVis) 29, 3 (2010), 1163-1172. 3

[FR94] FRIK T. F., RoshKo A.: Vortical structure in the wake of a transverse jet. Journal of Fluid Mechanics 279 (1994), 1-47. 8

[GGK*12] Grout R. W., Gruber A., Kolla H., Bremer P.-T., Bennett J. C., Gyulassy A., Chen J. H.: A direct numerical simulation study of turbulence and flame structure in transverse jets analysed in jet-trajectory based coordinates. Journal of Fluid Mechanics 706 (2012), 351-383. 1, 8

[GGTH07] Garth C., Gerhardt F., Tricoche X., Hagen H.: Efficient computation and visualization of coherent structures in fluid flow applications. IEEE Trans. on Vis. and Comp. Graph. 13, 6 (2007), 1464-1471. 2

[Gir88] GIRAULT V.: Incompressible finite element methods for Navier-Stokes equations with nonstandard boundary conditions in $\mathrm{R}^{3}$. Mathematics of Computations 51, 183 (1988), 55-74. 3

[Hal00] HALlER G.: Finding finite-time invariant manifolds in two-dimensional velocity fields. Chaos 10, 1 (2000), 99-108. 2,

[Hal01] HALLER G.: Lagrangian coherent structures and the rate of strain in two-dimensional turbulence. Physics of Fluids A 13 (2001), 3365-3385. 2, 3

[Hal05] Haller G.: An objective definition of a vortex. Journal of Fluid Mechanics 525 (2005), 1-26. 2

[Hel58] HeLmholtz H.: Über Integrale der hydrodynamischen Gleichungen, welche den Wirbelbewegungen entsprechen. Journal für die reine und angewandte Mathematik 1858, 55 (Jan 1858), 25-55. 3

[HH89] Helman J., Hesselink L.: Representation and display of vector field topology in fluid flow data sets. IEEE Computer 22,8 (1989), 27-36. 2

[Hun87] HUNT J.: Vorticity and vortex dynamics in complex turbulent flows. Trans. of the Canadian Society for Mechanical Engineering 11, 1 (1987), 21-35. 2, 7
[JH95] JEONG J., HuSSAIN F.: On the identification of a vortex Journal of Fluid Mechanics 285 (1995), 69-94. 2

[KB03] KumAR A., BARve S.: How and Why In Basic Mechanics. Universities Press, 2003. 4

[KRHH11] Kasten J., Reininghaus J., Hotz I., Hege H.C.: Two-dimensional time-dependent vortex regions based on the acceleration magnitude. IEEE Trans. on Vis. and Comp. Graph. 17, 12 (2011), 2080-2087. 2, 6

[Lug79] LugT H. J.: The dilemma of defining a vortex. In Recent Developments in Theoretical and Experimental Fluids Mechanics. Springer-Verlag, 1979, pp. 309-321. 2, 4

[MBP10] MALTRUd M., BRYAN F., PEACOCK S.: Boundary impulse response functions in a century-long eddying global ocean simulation. Environmental Fluid Mech. 10 (2010), 275-295.

[Nek13] Nektar++: http://www.nektar.info, 2013. 9

[PC94] PERRY A. E., CHONG M. S.: Topology of flow pattern in vortex motions and turbulence. Applied Scientific Research 53, 3-4 (1994), 357-374. 2

[PP03] POLTHIER K., PreUSS E.: Identifying vector fields singularities using a discrete Hodge decomposition. In Mathematical Visualization III (2003), Ed: H.C. Hege, K. Polthier, pp. 112134. 5

[PPF*10] Pobitzer A., Peikert R., Fuchs R., Schindler B., Kuhn A., Theisel H., Matkovic K., Hauser H.: On the way towards topology-based visualization of unsteady flow the state of the art. In EuroGraphics 2010 State of the Art Reports (STARs) (2010). 2

[PT84] PERRY A. E., TAN D. K. M.: Simple three-dimensional vortex motions in coflowing jets and wakes. Journal of Fluid Mechanics 141 (1984), 197-231. 2

[Sch95] Schwarz G.: Hodge Decomposition - A Method for Solving Boundary Value Problems. Springer Berlin, 1995. 5

[SLM05] Shadden S. C., LeKIEn F., Marsden J. E.: Definition and properties of Lagrangian coherent structures from finitetime Lyapunov exponents in two-dimensional aperiodic flows. Physica D 2012 (2005), 271-304. 3, 8

[Ste11] STEWART A. M.: Longitudinal and transverse components of a vector field. Sri Lankan Journal of Physics 12 (2011), $33-42.3$

[SWH05] SAHNER J., WEInKaUf T., HEgE H.-C.: Galilean invariant extraction and iconic representation of vortex core lines. In Proc. of Eurographics/IEEE VGTC Symp. on Visualization (EuroVis '05) (2005). 2

[vW02] VAN WIJK J. J.: Image based flow visualization. ACM Trans. on Graph. 21, 3 (2002), 745-754. 5

[WCW*11] Wiebel A., Chan R., Wolf C., Robitzki A., Stevens A., Scheuermann G.: Topological flow structures in a mathematical model for rotation-mediated cell aggregation. In Topological Methods in Data Analysis and Visualization. Springer Berlin Heidelberg, 2011, pp. 193-204. 3, 8

[WGS07] Wiebel A., Garth C., Scheuermann G.: Computation of localized flow for steady and unsteady vector fields and its applications. IEEE Trans. on Vis. and Comp. Graph. 13, 4 (2007), 641-651. 3

[YRSC11] Yoo C. S., RichaRdSON E. S., SANKARAN R., CHEN J. H.: A dns study on the stabilization mechanism of a turbulent lifted ethylene jet flame in highly-heated coflow. Proc. of the Combustion Institute 33, 1 (2011), 1619-1627. 1, 7 